\title{
A clinical audit to determine adherence in the Norfolk and Norwich University Hospital to the NICE guidelines for nutritional support in adults in the prevention and treatment of refeeding syndrome
}

\author{
S. E. Kimpton \\ Dietitians and Nutrition Team, Norfolk and Norwich University Hospital, Norwich NR4 7UY, UK
}

Refeeding syndrome results from severe fluid and electrolyte shifts in malnourished patients, and is associated with the initiation of nutritional support. ${ }^{(1)}$ NICE has categorised risk as: moderate, high and severely high. ${ }^{(2,3)}$ High risk patients are recommended an initial feed rate of $10 \mathrm{kcal} / \mathrm{kg} / \mathrm{day}$. A recent enquiry into the care of hospital patients receiving parenteral nutrition, found only a low number had their nutritional requirements documented. ${ }^{(4)}$

This audit aimed to determine the current practice in the prevention and treatment of refeeding syndrome in this Trust. Also the adherence to NICE guidelines and the use of the Malnutrition Universal Screening Tool (MUST).

The audit took place over 11 weeks. Patients initiated on artificial feeding were identified by either a dietitian or the nutrition team. The patients were assessed for inclusion criteria (over 18 years, started on artificial nutrition whilst an inpatient) and exclusion criteria (end-oflife treatment, supplementary artificial nutrition, artificial nutrition initiated whilst in the critical care complex). Data were collected for 7 days using the drug chart, observation chart, medical notes, dietetic chart and blood levels. A pilot study was conducted on one patient to assess the appropriateness of the data collection form.

37 patients were identified, 30 of these were found to be suitable. Of the 30 patients, $19(63.3 \%)$ were male (median age; 77.5 , range; 31-94). 19 patients $(63.3 \%)$ were receiving artificial nutrition due to an unsafe swallow, $6(20 \%)$ had undergone gastrointestinal surgery and $5(16.7 \%)$ were previously receiving inadequate oral intake. $16(53.3 \%)$ patients were documented as at risk of refeeding syndrome. 25 patients $(83.3 \%)$ had their nutrition managed by a dietitian, $11(36.7 \%)$ by the nutrition team and $1(3.3 \%)$ by their doctor, $8(26.7 \%)$ patients were managed by more than one healthcare professional. 20 patients $(66.7 \%)$ received nasogastric feeding, 4 (13.3\%) gastrostomy feeding, 1 nasojejual feeding and $5(16.7 \%)$ parenteral nutrition. Adherence to audit standards was obtained from the audit tool.

\begin{tabular}{lc}
\hline Standard & Adherence $(\%)$ \\
\hline MUST score calculated & 50 \\
Vitamin B started prior to feeding & 85 \\
No delay in starting feeding & 76.7 \\
Initial feed rate appropriate & 66.7 \\
Baseline blood levels & 56.7 \\
Adequate follow-up bloods & 33.3 \\
Corrections of electrolytes levels & 82.4 \\
\hline
\end{tabular}

This small audit recognizes identified areas of the management of refeeding syndrome that need further consideration. It would be useful to undertake this audit on a larger scale to substantiate these findings.

1. Solomon SM, Kirkby DF (1990) The refeeding syndrome: a review. J Parenteral Enteral Nutrition 14, 90-7.

2. National Institute for Health and Clinical Excellence. Nutritional support in adults, Oral nutrition support, enteral tube feeding and parenteral nutrition. Clinical guideline CG32, February 2006.

3. Tomlin G, et al. Guidelines for the management and prevention of the refeeding syndrome. Oxford Radcliffe Hospitals NHS trust, February 2009

4. Stewart JAD, Mason DG, Smith N, Protopapa K \& Mason M. A mixed bag: An enquiry into the care of hospital patients receiving parenteral nutrition. NCEPOD 2010. 